\title{
A framework using topological pathways for deeper analysis of transcriptome data
}

\author{
Yue Zhao ${ }^{\text {** }}$, Stephanie Piekos ${ }^{2}$, Tham H. Hoang ${ }^{1}$ and Dong-Guk Shin ${ }^{1 *}$
}

From 14th International Symposium on Bioinformatics Research and Applications (ISBRA'18)

Beijing, China. 8-11 June 2018

\begin{abstract}
Background: Pathway analysis is one of the later stage data analysis steps essential in interpreting high-throughput gene expression data. We propose a set of algorithms which given gene expression data can recognize which portion of sub-pathways are actively utilized in the biological system being studied. The degree of activation is measured by conditional probability of the input expression data based on the Bayesian Network model constructed from the topological pathway.

Results: We demonstrate the effectiveness of our pathway analysis method by conducting two case studies. The first one applies our method to a well-studied temporal microarray data set for the cell cycle using the KEGG Cell Cycle pathway. Our method closely reproduces the biological claims associated with the data sets, but unlike the original work ours can produce how pathway routes interact with each other above and beyond merely identifying which pathway routes are involved in the process. The second study applies the method to the p53 mutation microarray data to perform a comparative study.

Conclusions: We show that our method achieves comparable performance against all other pathway analysis systems included in this study in identifying p53 altered pathways. Our method could pave a new way of carrying out next generation pathway analysis.
\end{abstract}

Keywords: Topological Pathway Analysis, Bayesian Network, Depth First Search

\section{Background}

In this era of biomedical big data, a noticeable trend is that newly acquired genomics data (specifically, gene expression data) is compared with the prior known gene regulation relationships which are typically organized into curated molecular pathways (e.g., KEGG [1], Biocarta [2], Reactome [3], Wikipathways [4]). In general, gene expression data is first processed to identify significant differentially expressed (DE) genes using statistical methods like Limma [5], SAM [6], SPH [7], etc. These identified $\mathrm{DE}$ genes are then divided into groups of similar patterns

*Correspondence: yue.2.zhao@uconn.edu; dong.shin@uconn.edu ${ }^{1}$ Computer Science and Engineering Department, University of Connecticut, 371 Fairfield Way, Unit 4155, 06269 Storrs, USA

Full list of author information is available at the end of the article using clustering programs [8] or pattern based programs $[9,10]$. Each group of similarly behaving genes is then examined to test if each group includes genes known for any particular biological function (e.g., GOStat [11]) or molecular pathway at unusually high frequencies (e.g., DAVID [12], GSEA [13]). Although these gene enrichment analysis methods are useful in recognizing some basic nature of perturbed signals of the biological system under study, they do not discern if any specific pathway is activated or suppressed other than the fact that some pathway could be highly involved in the experimental system being studied. The next generation pathway analysis methods aimed at overcoming such deficiency of gene enrichment methods by organizing known gene-gene interaction relationships into topological pathways and 
analyze gene expression data on top of them so that the activated or suppressed state of the pathway can be computationally revealed (e.g., PARADIM [14], SPIA [15]).

We previously published topology-based pathway analysis methods belonging to this next generation pathway analysis system [16-19]. Specifically, our method presented in $[18,19]$ departs from the conventional topologybased systems like PARADIM or SPIA in the sense that our method dynamically encodes pathway routes as a Bayesian network and uses both gene expression and mutation data as input and identifies not only if any pathway is activated or suppressed but also through which route(s) of the pathway such gene expression perturbation could be propagating. However, one limitation of our previous work is that the method requires preselection of the start and end of pathway routes to be analyzed. In addition, through empirical studies, we discover that our previous method tends to identify "choppy" pathway routes that are partially activated or suppressed, thus less useful if one's goal is to find overall patterns of pathway route usages. The goal of this paper is to report the extension of our previous work $[18,19]$ in which multiple new algorithms are introduced to isolate highly regulating (activation and/or suppression) sub-components of the pathways and conveniently visualize the overall patterns of pathway activation or suppression directly over the pathway diagrams. We call this system Deep Pathway Analyzer (DPA).

Among existing gene set enrichment analysis methods, GSEA is one of the most popular software packages in which computing the enrichment score is done by a variation of the weighted Kolmogorov-Smirnov-like statistic [13]. SPIA by [15] is a topology-based system and it proposes to measure pathway significance by performing statistical tests against random permutation. An improvement over SPIA is PARADIGM [14] which models the pathway as a factor graph and uses a statistical method to compute a sample specific inference, specifically for genomics data obtained from cancer patients. Two recent systems by [20] and [21] also encode the pathway as a Bayesian network. After removing cycles in the graph, they train the model with expression data. Significance of the score is produced by bootstrap-generated data. DRAGEN by [22] detects differentially expressing genes by performing a hypothesis testing designed to figure out if linear model has identical parameters. Most recently, Altered Pathway Analysis tool (APA) by [23] aims to detect altered pathways by dynamically calculating pathway rewiring through analyzing correlation between genes, but this system does not use prior knowledge. Our work is different from these existing topology-based systems by the feature, what we call, route-based recognition capability, and using this feature we can produce deeper analysis outcomes suggesting how identified "perturbed" pathway routes may interact with each other.

The rest of this paper is organized as follows. "Methods" section briefly reviews existing pathway analysis methods and introduces the Bayesian network model and the algorithms newly developed. Section III describes the results of our algorithm being tested using a public domain temporal microarray data set from the cell cycle experiment [24]. Afterwards we show the outcome of applying our algorithm to the p53 mutation microarray data and specifically compare our analysis outcome with the similar analysis done by [23]. Two case studies are shown to demonstrate the generality of our enhanced method. Lastly, Section VI is the conclusion.

\section{Methods}

In this section we first briefly review the methodology proposed in our previous work [19] for the sake of completeness and then present two new algorithms that are designed to improve deficiencies of the earlier system.

\section{Review of the previous Model}

The key idea of DPA is identifying "routes" of aberrant pathways. Each pathway route $G^{*}$ is encoded as a Bayesian Network $G$ which is initialized with a sequence of conditional probabilities which are designed to encode directionality of regulatory relationships encoded in the pathways, i.e., activation and inhibition relationships. The transformation process from $G^{*}$ to the corresponding Bayesian Network $G$ is illustrated in Algorithm.1. Next we show the biological interpretation logic behind the conditional probability table for $e_{i j}$. Consider the activation table given in (Table.1) (for the inhibition table, refer to (Table 2) which is built in a similar way): If the parent gene of $g_{j}, g_{i}$, has function gain mutation, and overly expressed, namely $M_{i}=R_{i}=+1$, then the target $g_{j}$ would also be highly likely to overexpress, i.e. $R_{j}=+1$, given the edge between them in $G^{*}$ is 'activation. As a result,

$$
P\left(R_{j}=+1 \mid M_{i}=R_{i}=+1\right)=1-\epsilon
$$

where $\epsilon$ is the error rate we can tolerate and is close to zero. Similarly, if the parent gene of $g_{j}$ has function loss mutation, or its expression level is down-regulated in test case, then the downstream regulation towards $g_{j}$ would be likely not functioning. Therefore, $g_{j}$ would tend to be

Table 1 Activation

\begin{tabular}{llll}
\hline$M_{i}$ & $R_{i}$ & $R_{j}=+1$ & $R_{j}=-1$ \\
\hline+1 & +1 & $1-\epsilon^{*}$ & $\epsilon$ \\
-1 & +1 & $\gamma$ & $1-\gamma$ \\
+1 & -1 & $1-\gamma$ & $\gamma$ \\
-1 & -1 & $\epsilon$ & $1-\epsilon$ \\
\hline
\end{tabular}


Table 2 Inhibition

\begin{tabular}{llll}
\hline$M_{i}$ & $R_{i}$ & $R_{j}=+1$ & $R_{j}=-1$ \\
\hline+1 & +1 & $\epsilon$ & $1-\epsilon$ \\
-1 & +1 & $1-\gamma$ & $\gamma$ \\
+1 & -1 & $\gamma$ & $1-\gamma$ \\
-1 & -1 & $1-\epsilon$ & $\epsilon$ \\
\hline
\end{tabular}

${ }^{*} \epsilon \in(0,0.5)$ is the error rate we could tolerate

underexpressive, namely $R_{j}=-1$, and the corresponding probability is flipped.

Let the pathway of interest be converted into a gene regulation network $G_{B}=\left(V_{B}, E_{B}\right)$, where $V_{B}=\left\{g_{i} \mid i=\right.$ $\left.1 \ldots\left|V_{B}\right|\right\}$ and $E_{B}=\left\{\left(g_{i}, g_{j}\right) \mid g_{i}, g_{j} \in V_{B}\right\}$. Consider a given pathway route $G^{*}=\left(V^{*}, E^{*}\right)$ in $G_{B}$ where $V^{*}=\left\{g_{i_{k}} \mid k=\right.$ $\left.1 \ldots\left|V^{*}\right|\right\}$ and $E^{*}=\left\{\left(g_{i}, g_{j}\right) \mid g_{i}, g_{j} \in V^{*}\right\} \subset E_{B}$.

Once the Bayesian Network $G$ is generated from $G^{*}$, the pathway route is ranked by conditional probability of the observed data given $G$ normalized by $P(\mathbf{R}, \mathbf{M}$ are consistent $\mid G)$ as shown in (1) [19] where $\mathbf{r}_{s}$, $\mathbf{m}_{s}$ are, respectively, the expression observation and the mutation observation for the sample $s$.

Advantages of this measure are: (i) the analysis could allow biologists to easily pinpoint which biological processes are likely to be overly activated or suppressed; and (ii) even though some expression values are flipped due to random errors from the genomic data (it is observed to be -1 when it is actually +1 ), the whole path would still have a high score since the majority of other genes could have consistent expression observations.

$$
\operatorname{Score}\left(G^{*}, \mathbf{r}_{s}, \mathbf{m}_{s}\right)=\frac{P\left(\mathbf{R}=\mathbf{r}_{s}, \mathbf{M}=\mathbf{m}_{s} \mid G\right)}{P(\mathbf{R}, \mathbf{M} \text { are consistent } \mid G)}
$$

\section{THE REGULATION PROCESS FOR $e_{i j}$ IN $G^{*}$}

Then the score is extended to be a signed score by (2) which varies from -1 (highly suppressed) to +1 (highly enhanced). The definition of a pathway route being "activated" or "suppressed" is the following.

$$
\begin{aligned}
\operatorname{sScore}\left(G^{*}, \mathbf{r}_{s}, \mathbf{m}_{s}\right) & =\tilde{I}\left(r_{s}^{\left|G^{*}\right|}, \dot{r_{s}}\left|G^{*}\right|\right) \cdot \operatorname{Score}\left(G^{*}, \mathbf{r}_{s}, \mathbf{m}_{s}\right) \\
\tilde{I}(x, y) & = \begin{cases}+1 & x=y \\
-1 & x \neq y\end{cases}
\end{aligned}
$$

where $r_{s}^{\left|G^{*}\right|}$ is the observed expression level of the last available node for the input sample $s$ in the route $G^{*}$ and $\dot{r}_{s}^{\left|G^{*}\right|}$ is the expected expression level of the same node calculated by the interpretation logic.

Aggregating the scores for routes in a pathway, we define the pathway score in (3). We simply measure the significance of this pathway, $G_{B}$, by using the proportion of routes that have an average of all the patients' scores, calculated by equation (1), that is larger than some threshold $t$. Each perturbed route is weighted by its length.

$$
\begin{aligned}
\operatorname{pScore}_{S}\left(G_{B}\right)= & \frac{1}{\sum_{G^{*} \in G_{B}} w_{G^{*}}} \\
& \sum_{G^{*} \in G_{B}} w_{G^{*}} I\left(\frac{1}{|S|} \sum_{s \in S} \operatorname{Score}_{S}\left(G^{*}\right) \geq t\right)
\end{aligned}
$$

\section{Statistical Significance Measure on the Route Score}

In this section, we introduce a new measure to quantify the statistical significance for the route score: the probability of route score being one in (1) conditioning on the observation for each gene in route $G^{*}$ being randomly generated. The formula is shown in (4). Mutation data $m_{s}$ is sparse and the probability of observing given $m_{s}$ by chance is close to zero, thus it is not proper to consider the randomness of $m_{s}$ here. Based on this assumption, $m_{s}$ is treated as prior parameter. Thus the score is reduced to (5).

$$
\begin{aligned}
\operatorname{SigS\operatorname {Scre}}\left(G^{*}, \mathbf{r}_{s}, \mathbf{m}_{s}\right) & =P\left(\mathbf{r}_{s}, \mathbf{m}_{s} \text { are consistent } \mid P_{0}\right) \\
P_{0}: R & = \begin{cases}+1 & p=0.5 \\
-1 & p=0.5\end{cases}
\end{aligned}
$$

$\operatorname{SigS\operatorname {Score}}\left(G^{*}, \mathbf{r}_{s}, \mathbf{m}_{s}\right)=P\left(\mathbf{r}_{s}\right.$ are consistent $\left.\mid P_{0}, \mathbf{M}_{s}=\mathbf{m}_{s}\right)$

Suppose $w$ is the number of genes in the route, then

$\operatorname{SigS\operatorname {core}}\left(G^{*}, \mathbf{r}_{s}, \mathbf{m}_{s}\right)=P\left(\mathbf{r}_{s}\right.$ are consistent $\left.\mid P_{0}, \mathbf{M}_{s}=\mathbf{m}_{s}\right)$

$$
=2(0.5)^{w}=(0.5)^{w-1}
$$

In order to measure the significance of the pathway score in (3), we calculate the probability of observing $Q$ differentially regulated routes in a pathway $G_{B}$ given the observations are selected randomly. The number $Q$ follows Poisson Binomial Distribution [25] and this probability can be approximated by (6) [26] assuming $G_{B}$ consists of $k$ routes.

$$
\begin{aligned}
\text { SigScore }_{G_{B}}=\operatorname{Pr}(Q=q) & \approx \operatorname{Binom}\left(n, \frac{\mu}{k}\right) \\
\mu & =\sum_{G^{*} \in G_{B}} \prod_{s} \operatorname{SigScore}\left(G^{*}, \mathbf{r}_{s}, \mathbf{m}_{s}\right)
\end{aligned}
$$

This probability can serve as the p-value of the hypothesis test whose null hypothesis is that the observation is generated randomly by $P_{0}$. Thus low SigScore $_{G_{B}}$ indicates rejection of null hypothesis, and the lower the SigScore is, the more significant the calculated pathway score is.

Hyper Parameter Analysis and Dynamic Parameter Setting In this section, we discuss issues related to setting the hyper-parameters. The key idea behind setting the hyper parameters is to make the false discovery rate associated 
with route score controllable. Consider a pathway route $G^{*}$ with length of $\left|G^{*}\right|$ having $\left|G^{*}\right|-1$ edges. The route score in (4) can be approximated by a simpler formula (7) involving the number of inconsistent edges, $K$. This formula is to capture the intuition that whenever an inconsistent edge is discovered according to data, we penalize the score by the hyper-parameter $\epsilon$, and otherwise we reward the score by $1-\epsilon$.

$$
\operatorname{SigScore}\left(G^{*}, K\right)=\frac{\epsilon^{K}(1-\epsilon)^{\left|G^{*}\right|-1-K}}{(1-\epsilon)^{\left|G^{*}\right|-1}}=\left(\frac{\epsilon}{1-\epsilon}\right)^{K}
$$

Next we proceed to derive the distribution of $K$ assuming that each edge is discovered inconsistent independently by chance. That is, for each edge $e_{i}, i=1 \ldots\left|G^{*}\right|-$ 1 , we define Bernoulli random variable $X_{i}=1$ if $e_{i}$ is inconsistent; $X_{i}=0$ otherwise. Thus $P\left(X_{i}=1\right)=$ 0.5, $X_{i}$ i.i.d. $\sim$ Bernoulli(0.5). Then $K=\sum_{i} X_{i}$ follows Binomial distribution $\operatorname{Bin}\left(0.5,\left|G^{*}\right|-1\right)$ since $X_{i}$ 's are independent. Based on this distribution, one can pick $\epsilon$ such that the majority of the scores generated under null hypothesis (the portion $\geq 1-\alpha$ ) is less than some threshold $t$, namely, $P\left(\operatorname{SigScore}\left(G^{*}, K\right) \geq t\right)<\alpha$. Since SigScore in (7) is monotonically decreasing by $K$, we have

$$
\begin{aligned}
& P\left(\left(\frac{\epsilon}{1-\epsilon}\right)^{K} \geq t\right)<\alpha \\
& P\left(K \leq\left(\log \frac{\epsilon}{1-\epsilon}\right)^{-1} \log t\right)<\alpha \quad \forall \epsilon \in(0,0.5)
\end{aligned}
$$

Since $K$ 's distribution is known, we can assign $\left(\log \frac{\epsilon}{1-\epsilon}\right)^{-1} \log t$ to be no larger than $q_{\alpha}$, which is exactly the quantile value such that $P\left(K \leq q_{\alpha}\right)=\alpha$ as shown in Fig. 1. The quantile value is available from the binomial probability table. Actually since binomial distribution offers limited confidence level options, one can use the quantile of the normal approximation of the binomial distribution instead [27]. By solving the equation, we have:

$$
\left(\log \frac{\epsilon}{1-\epsilon}\right)^{-1} \log t \leq q_{\alpha}
$$

Here when $t=1, K \equiv 0$ (entirely consistent) should hold according to the equation (8) since $\epsilon \in(0,0.5)$. This indicates that independent of $\epsilon$, only routes with no inconsistency are discovered if $t=1$, but this arrangement would be too strict.

In case when $t \in(0,1)$,

$$
\epsilon \leq B(t, \alpha)=1-\frac{1}{1+\sqrt[q \alpha]{t}} \in(0,0.5)
$$

The intuition behind this formula is the following. The formula (9) clearly indicates that the upper bound of $\epsilon$, $B(t, \alpha)$, increases if either $t$ or $\alpha$ increases. If we keep the upper bound fixed, increasing $t$ will make $\alpha$ decrease while providing a better confidence level and thus resulting in a smaller false positive rate.
As far as the hyper-parameter $\gamma$ is concerned, given any edge $e_{i j}$ in the route, the marginal probability $P\left(R_{j}=\right.$ $\left.+1 \mid R_{i}=+1\right)=\sum_{M_{i}} P\left(R_{j}=+1 \mid R_{i}=+1, M_{i}\right) / P\left(R_{i}=\right.$ $+1)=(1+\gamma-\epsilon) / 2$ if no mutation information is available. To penalize inconsistency, one can set $\gamma$ larger than $\epsilon$. However, the inconsistency should also be penalized if mutation information is present as shown in Table 1-2, and in that case $\gamma$ decreases making $\gamma \in(\epsilon, 0.5)$. This also explains why the setting $\epsilon=0.1$ and $\gamma=0.25$ gives a good result as such outcome has been presented in one of our previous works [18]. In this paper, we choose to set $\gamma$ to be the midpoint between $\epsilon$ and 0.5 , namely, $(\epsilon+0.5) / 2$.

For all the experiments in this work, we dynamically calculate $\epsilon$ using the upper bound provided by (9) with threshold $t=0.8$ and $\alpha=0.05$ so that at most $5 \%$ scores generated randomly under the null hypothesis can become larger than 0.8 as such condition is guaranteed by (8).

\section{Algorithmic Approach to Deeper Pathway Analysis}

Here we propose a set of algorithms which aim to recognize all the "perturbed" portion of a pathway based upon input omics data which may include not only gene expression data but also mutation data. We label "perturbed" portion as the sub-network whose gene-gene interaction relationships are recognized as "perturbed" within the network topology when the input expression data is compared to the known relationships captured in the pathway network. It uses a Depth-First-Search[28] to extract all possible routes starting from a given node in the pathway and calculates the signed score using (2) at each step so that the perturbed portion, i.e., the subnetworks falling outsides of some threshold scores close to +1 for "activated" ( -1 for "suppressed") can be isolated. This process is described formally in Algorithm.2. Due to space limitation, only the algorithm calculating the "activated" portion of the pathway is shown. Identifying the suppressed portion of the pathways can be obtained by replacing line 5 in Algorithm.2 with 'if $r_{\left|G^{*}\right|}==-\dot{r}_{\left|G^{*}\right|}$ AND score $==1$ '.

The motivations for developing the algorithm are manifold. First, the route computation of a given pathway can be done dynamically. Second, this dynamic route computation and the generation of Bayesian network realtime allows performing the analysis comprehensively but efficiently because all small sub-segments of each long pathway route are examined independently and checked if any of sub-segments exceed the thresholds for determining significantly "activated" or "suppressed". Third, our algorithm solution is conducive to running the analysis in parallel for speed up. The complexity of examining all possible pathway sub-segments by running process GetRoutes with all existing nodes in the pathway $\left(v_{i}, i=\right.$ $\left.1 \ldots\left|V_{B}\right|\right)$ as starting node is exponential. But since examining each possible starting node in a given pathway is 


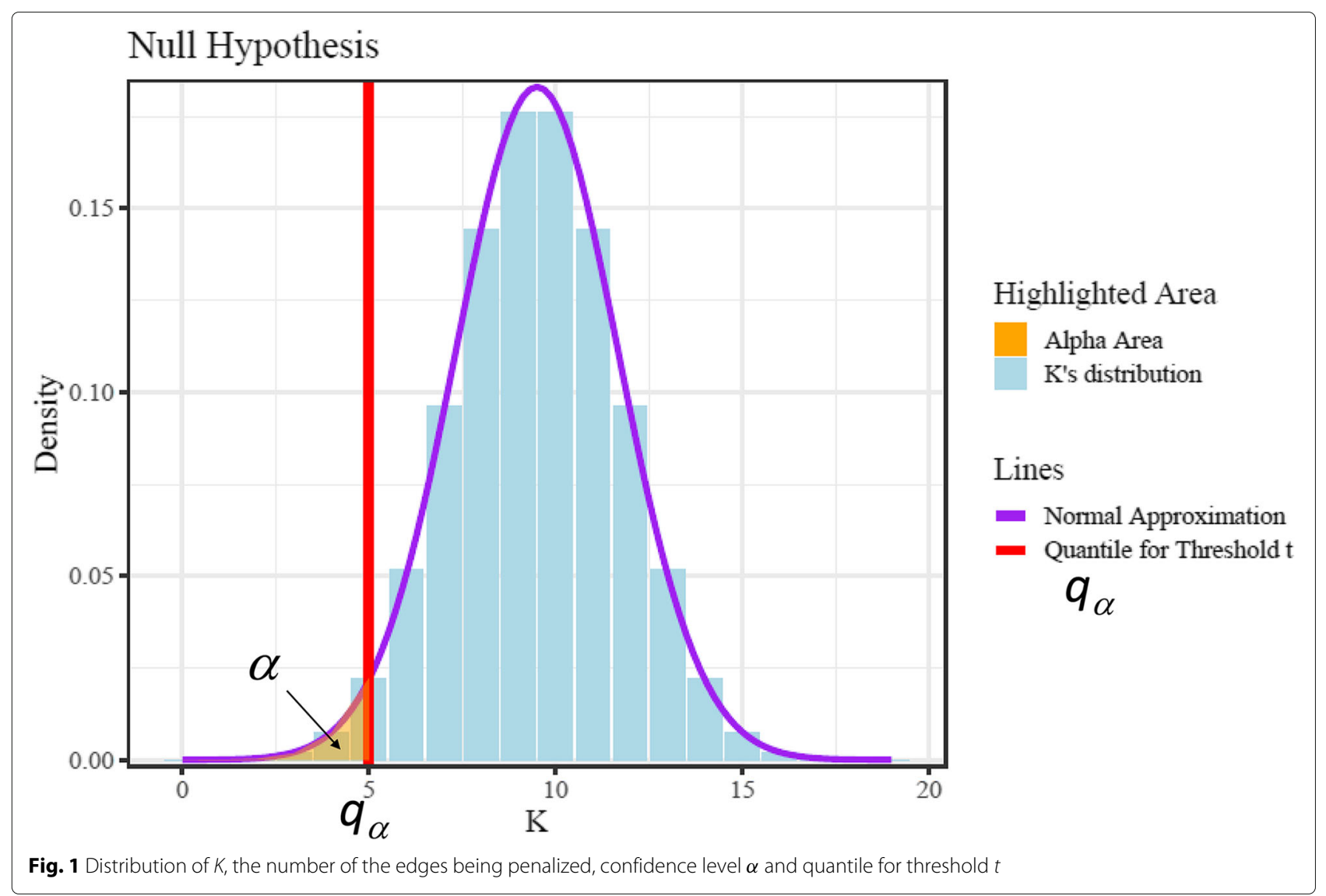

independent of each other, the $\left|V_{B}\right|$ processes can be easily parallelized. The time complexity of this algorithm is analyzed briefly. Since the algorithm runs depth first search through the pathway graph $G_{B}$, this takes $O(|E|)$ steps. For each step, we need to build the Bayesian Network and calculate the conditional probability (4). For $n$ random variables each possibly having $d$ different possible values, the calculation takes $O\left(d^{n}\right)$ in the worst case. However, in our application, our route setting makes $d \leq 2$ and $n \leq 3$ for each edge, meaning at most 3 nodes $\left(R_{i}, M_{i}\right.$ and $R_{j}$ for $\left.e_{i j}\right)$ each having at most 2 possible values are considered. In this case, calculating the probability takes $O(1)$ time. In summary, the algorithm takes linear time $O(|E|)$.

\section{Results}

\section{Cell Cycle Study}

Our first experiment is to apply our algorithms to the microarray data set by [24] which aimed to compare the gene expression pattern of well-publicized cell cycling phases, G1, S, G2, and M. Our method shows - for the first time - how the involved genes are interacting with each other in each phase over the pathway topology and how that interacting pattern changes over time revealing the repeating pattern of cell cycling phases.

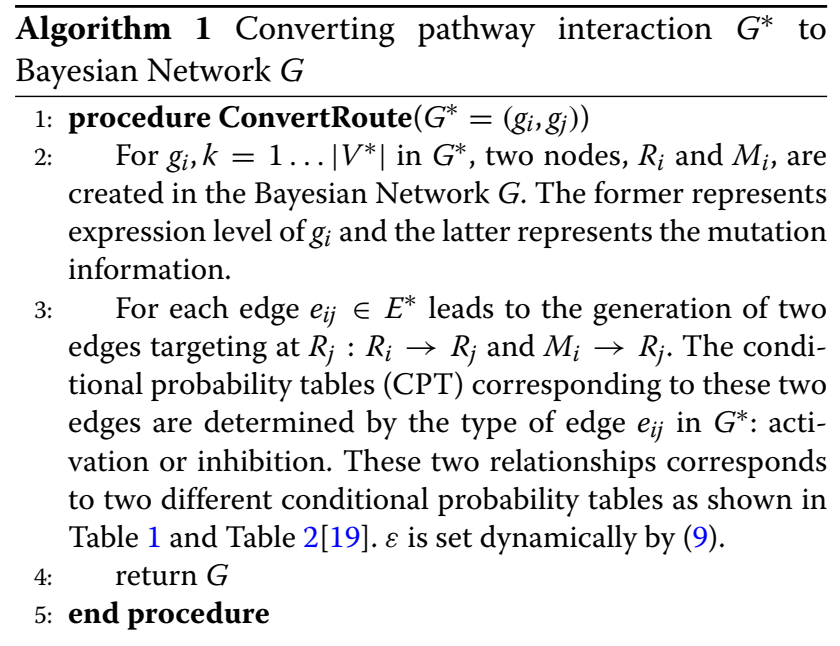

\section{Data Description}

The cell cycle data set by [24] used synchronized HeLa S3 cells. The microarray data was processed and log2 test-over-control RNA expression ratio was provided by the authors. We transformed the $\log 2$ ratios into expression observation $\mathbf{r}$ by (10). Log2 (Cy5/Cy3) was retrieved for each data point and used for all analyses, where 
$(\mathrm{Cy} 5 / \mathrm{Cy} 3)$ is the normalized ratio of the backgroundcorrected intensities, as defined in [29]. Algorithm.2 is run with input $G_{B}$ : KEGG Cell Cycle pathway gene regulation network and $D=(\mathbf{r}, \mathbf{m})$. Since no mutation information is available for the $\mathrm{S} 3 \mathrm{HeLa}$ cell, $\mathbf{m}$ is set as a null vector. The procedure is run on all possible starting nodes in $G_{B}$.

$$
r_{i}= \begin{cases}+1 & \log _{2}\left(\frac{C y 5_{i}}{C y_{i}}\right)>0 \\ -1 & \log _{2}\left(\frac{C y 5_{i}}{C y 3_{i}}\right)<0 \\ \text { missing } & \text { otherwise }\end{cases}
$$

\section{Result and Discussion}

After extracting all possible pathway routes from KEGG Cell Cycle pathway, we calculate the scores at each time point. The network diagram shown in Fig. 2 is the Cell Cycle pathway from KEGG in which genes are displayed as nodes and prior known relationships of activation or inhibition are shown as directed edges. One important observation from this network diagram is that the changes of perturbed patterns closely match the anticipated transition of four cell cycling phases of G1, S, G2, and M as reported in the literature. The human cell cycle is a finely-tuned regulatory system consisting of multiple cellular checkpoints that allow the cell to progress through each phase, ensuring proper division. A network of proteins such as cyclins (CCNs), cyclin-dependent kinases (CDKs), and CDK inhibitors (CDKNs) regulate the cell's transition into each phase. Changes in gene expression at the transcriptional level can be seen throughout the cell cycle, with certain genes being expressed temporally at either higher or lower levels depending on the phase of the cycle the cell is in [31]. According to the literature, mRNA levels of most of these genes correlate with their function [32]. The patterns presented in Fig. 2 could be regarded as "signatures" of pathways at different time points during the cell cycle.

Next is to report that the route scoring scheme presented by (1) successfully captures the information for each cell cycle phase. A multinomial regression LASSO model [33] is fitted to predict each cell cycle phase given the route scores calculated at different time points. By setting the penalty coefficient of 0.32 , we compute the top features for each phase and the result is shown in Table 3.

Although the Cell Cycle pathway shown in Fig. 2 is from KEGG, it was rendered into a network to emphasize its repeating patterns using Rgraphviz package. Since scientists who use KEGG graphs are not familiar with this rendering, we show in Fig. 3 the original KEGG Cell Cycle graph with routes identified in Table 3 for each cell cycle annotated in different colors, purple for G1, blue for S, yellow for $\mathrm{G} 2$ and orange for $\mathrm{M}$. What is noticeable in this color coded display over the original KEGG Cell Cycle graph is that all four routes for G1 through M phases

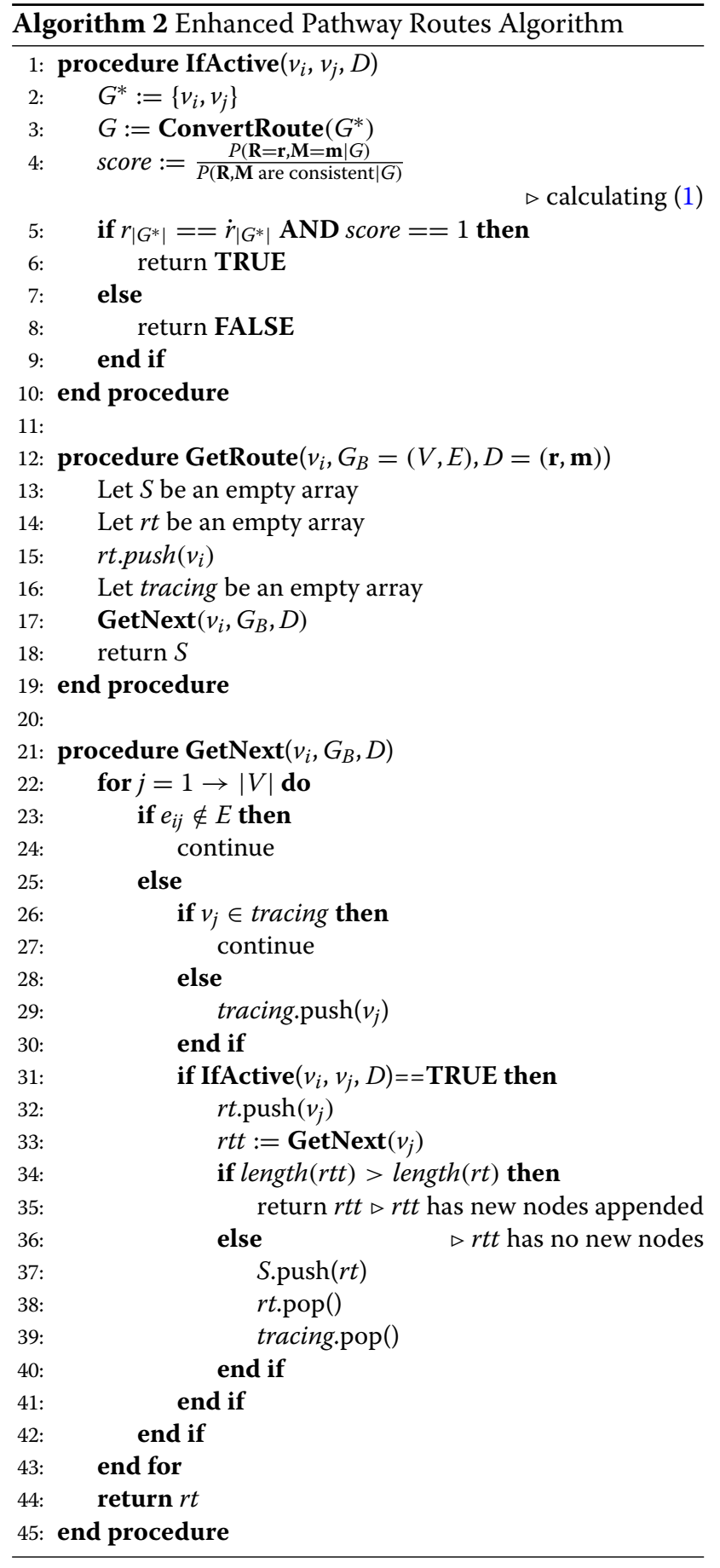

clearly coincide with the nodes mapped by their respective colors. What is also noticeable in this figure is that the color coding of four routes (G1, S, G2 and M) approximately reveal their respective positions from left to right of the graph. This pattern clearly matches that this particular KEGG graph is designed to show the transition of G1 through M left to right as such temporality is actually annotated at the bottom of the original graph. We also 


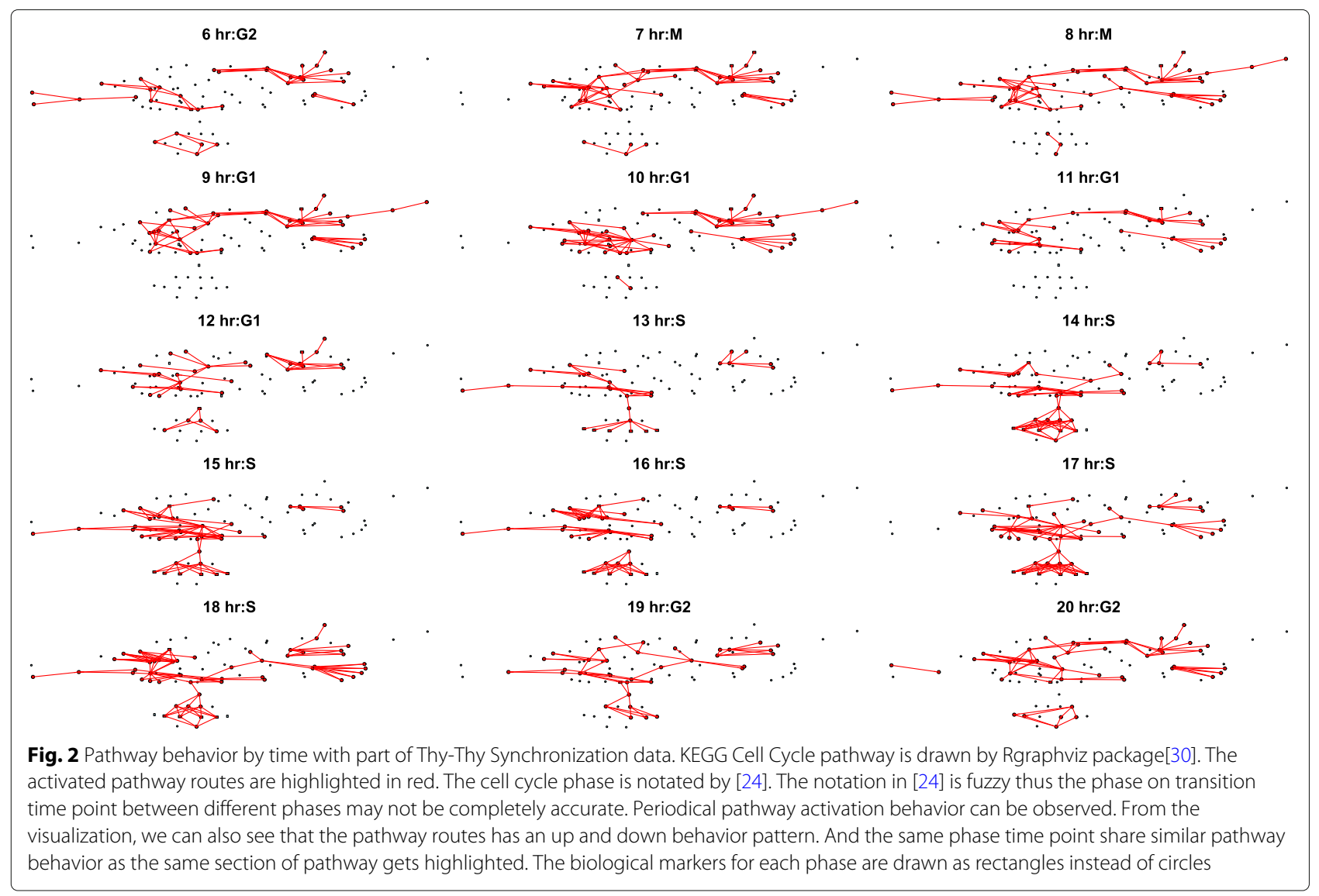

note that the yellow and orange color overlay of routes for G2 and M phases lands almost at the same set of nodes. This is expected since the two phases are usually not separable and for that reason they are generally denoted as G2/M phase[24].

Lastly, we show the result of performing hierarchical clustering on the identified routes and scores in Fig. 4a. Noticeable in this heat map is the clear consistency between the transition of cell cycling phases and what has been reported in the original publication of the data set [24].

\section{Comparison with Other Tools on P53 Mutation Dataset}

In this section, same pathway analysis [23] on p53 mutation dataset[34] is performed with Deep pathway analysis (DPA). The corresponding results is then compared

Table 3 Top features selected for each cell cycle phase

\begin{tabular}{lll}
\hline$\beta$ & Routes & Class \\
\hline 0.5596 & BUB1, BUB3, ANAPC10, PTTG2, ESPL1, STAG1 & $\mathrm{M}$ \\
0.3688 & TFDP1, CCNE1, RB1 & $\mathrm{S}$ \\
0.3222 & BUB1, BUB3, ANAPC10, PTTG2, ESPL1, SMC1A & G2 \\
0.0894 & CUL1, CDKN1A, CDK6 & G1 \\
\hline
\end{tabular}

against the APA[23], ORA[35], GSCA[36], GSNCA[37], ESEA[38], SPIA[15], PWEA[39] and DRAGEN[22].

\section{Data Description}

The p53 mutation microarray dataset has been widely used as a pathway enrichment analysis benchmark, containing 33 test samples having mutated p53 and 17 wild type control samples. First, the test vs control data is processed with LIMMA[5] using the same parameter settings as [23] and the detected significantly (LIMMA p-value $<$ 0.05) Differentially Expressed(DE) genes $\log F C$ score is used as $\mathbf{r}$ data for DPA. Since TP53 is known mutated in this sample, we set $m=-1$ for TP53 and $m=$ NULL for all others. We attempted to follow the exact same procedure used for the pathway analysis described in [23] as our objective was to make a direct comparison between APA [23] and our work DPA. The pathways having at least one target gene of p53 is labeled as 1 and the other pathways are labeled as 0 where the targets of p53 are obtained from [40]. Unfortunately, both KEGG pathway database and p53 target gene list have been updated since APA has been published. Thus we only used 148 pathways of which 66 labeled as 1 and limited our comparison against the proportion of pathways correctly predicted in [23]. 


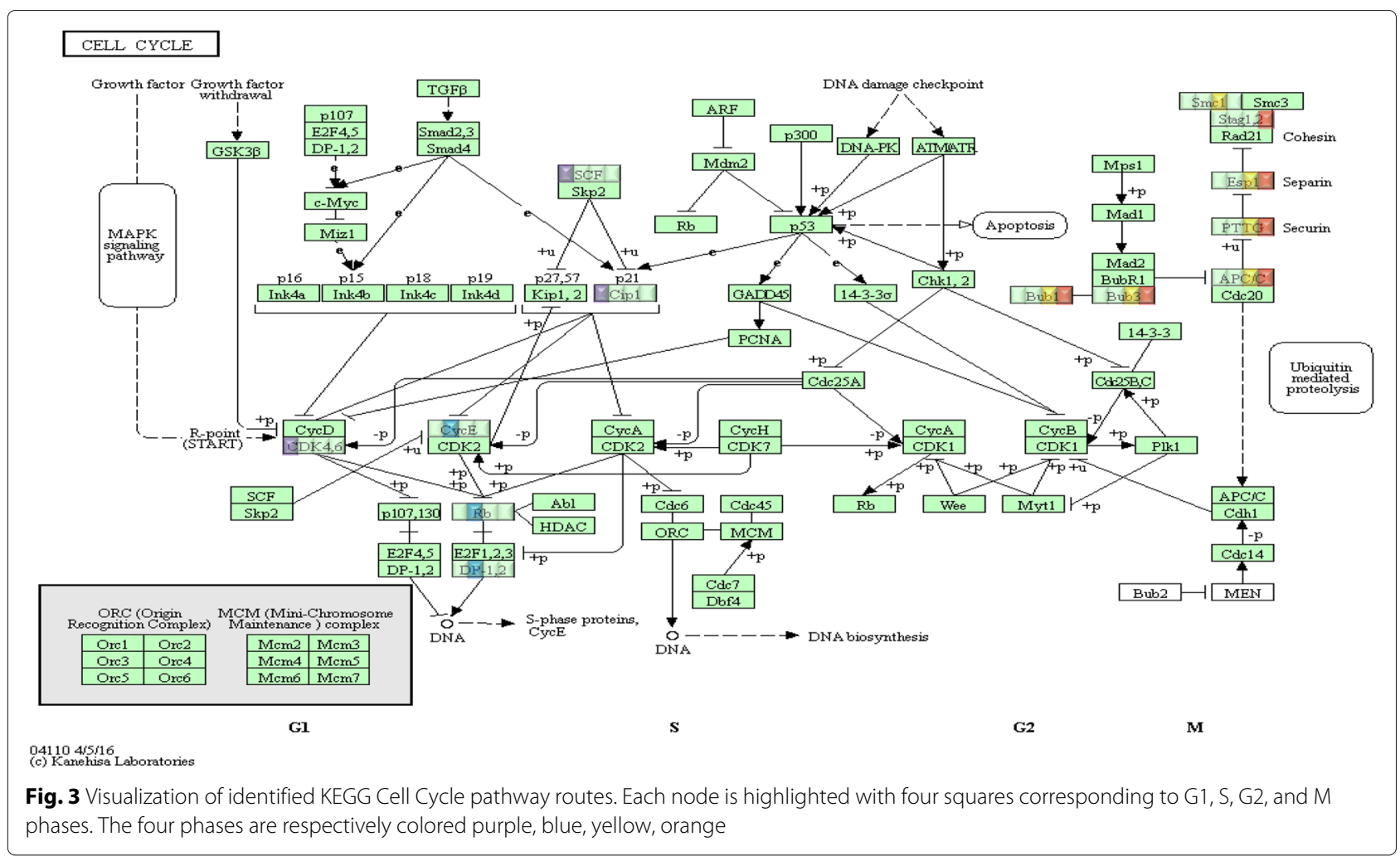

\section{Result and Discussion}

We use the pathway score, $p S c o r e$, given in (3) to rank the pathways. We calculate the true positive rate and false positive rate in which pathways with scores higher than some threshold is declared to be class 1. By taking different thresholds, ROC curve is obtained and the result is shown in Fig. 4b. The best Area Under Curve (AUC) for DPA is 0.78 and this value is close to 0.8 which has been reported for APA [23]. The proportion of correctly predicted "altered" pathways for each study is shown in Fig. 4c. In this figure, the data for ORA, ESEA, GSNCA, PWEA, SPIA, DRAGEN and APA are directly imported from [23] and included for the comparison purpose. Noticeable in this figure is that DPA reports higher percentage of p53 altered pathways than APA (i.e., 0.80 vs. 0.75 ). Specifically, DPA predicted 53 out of 66 $(0.80)$ as altered pathways where key known ones such as "Pathways in cancer", "Jak-STAT signaling pathway", "Prostate Cancer pathway", and "p53 signaling pathway" are all included. We note that there are 61 pathways identified as altered by both APA $(D R \geq 0.05)$ and DPA (pScore $>0)$. In that regard, the accuracies of both systems can be seen quite comparable. As an alternative comparison study, APA and DPA have been compared using only 132 pathways from the newest version of KEGG, 66 for class 1 (i.e., containing p53 targets) and 66 for class 0 (i.e., containing no p53 target). The results are 0.76 for
APA and 0.78 for DPA making DPA outperforms APA by 0.02 .

In terms of delivering explanation for the biologists, DPA offers a far greater benefit over APA by presenting the prior knowledge in a manner that biologists are familiar with, i.e., gene regulatory relationships organized into topological pathways. One reason that previous pathway analysis tools fails to work well is because they mainly try to discover perturbed pathways by individual differentially expressed genes instead of gene to gene interactions [23]. Both APA and DPA are newer generation pathway analysis systems which exploits gene-gene interaction relationships in calculating the degree of pathway perturbation, but there is one major difference between APA and DPA in the mechanisms of identifying altered pathways. APA constructs pathway networks dynamically based on gene co-expression whereas DPA uses activation relationship and inhibition relationship as two different forms of prior knowledge. APA measures the perturbation in a pathway by the "similarity" between gene expression test data and control data but DPA measures the "consistency" between expression data and the regulatory relationships encoded into the pathway diagrams.

Lastly, to show the effectiveness of SigScore given in (6), we calculate the $-\log$ (SigScore) for false positive (pathways not having p53 targets but identified) vs true positive (pathways having p53 and identified) and 


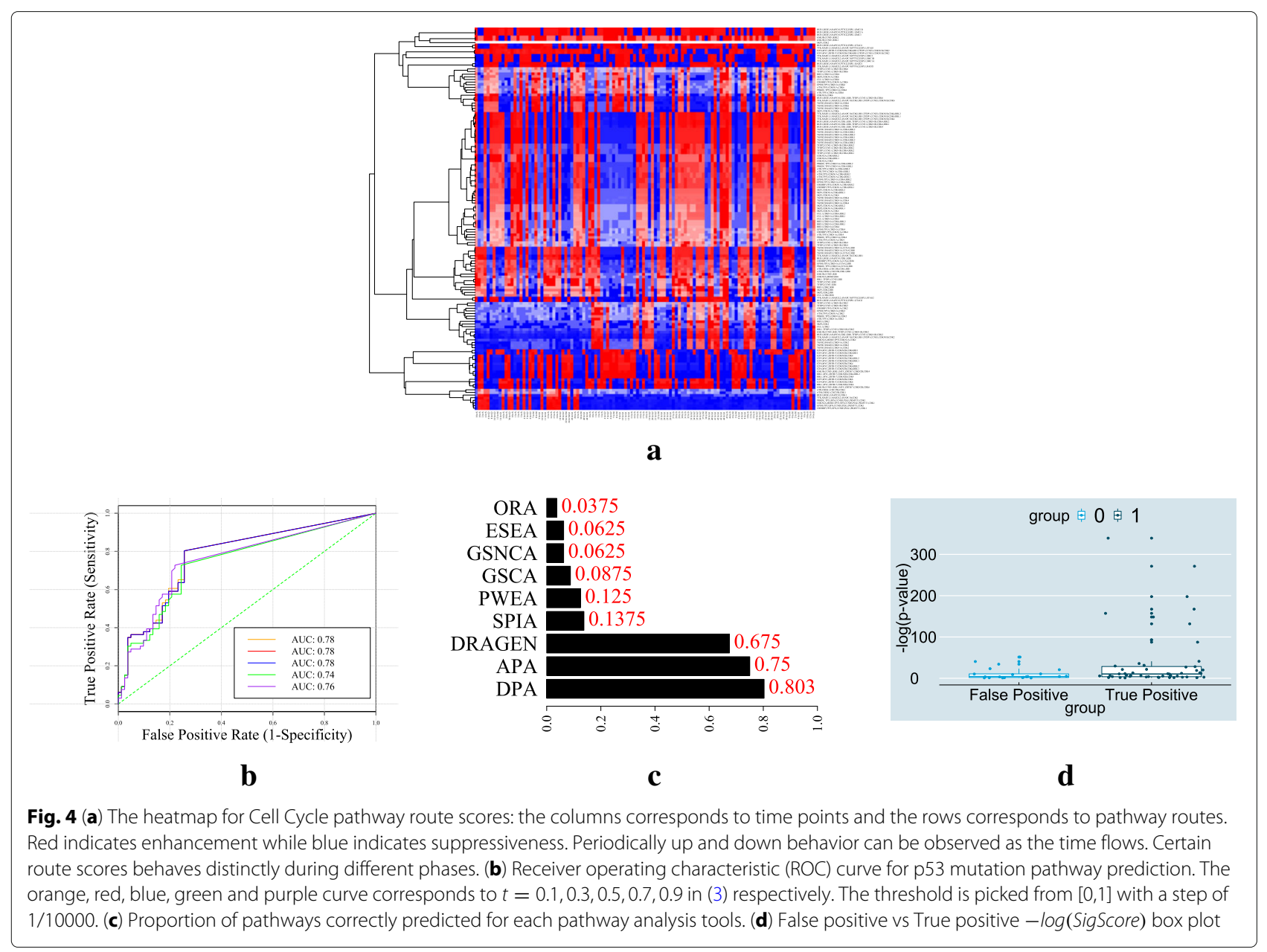

produce a box plot comparison, as shown in Fig. $4 \mathrm{~d}$. The Welch two sample $t$-test[41] performed for these two groups of SigScores produces the p-value 0.008634, clearly suggesting that the SigScores for the true positive group are significantly lower than those for the false positive group. This result indicates that SigScore can recognize pathways that acquire a high score by chance.

\section{Conclusions}

We proposed a set of algorithms which given a gene expression data set can compute and score the "perturbed" portion of biological pathways. This method identifies overly regulating routes (or "axes") of pathways by calculating the conditional probabilities of regulatory relationships encoded into Bayesian networks which are constructed from known biological pathways. Our method has been tested with two well-known, publicly available microarray data sets. In our application to the cell cycling microarray data, our method can "recognize" specific portions of pathways clearly revealing cell cycle phase transition with which biologists can easily identify the localized perturbation patterns. We demonstrated through pathway network visualization that our method can clearly reveal how activated pathway routes changes over time and if such pattern change repeats as cell cycling progresses. In our comparison study with APA, our approach demonstrates a comparable accuracy in recognizing perturbed pathways as our method algorithmically identifies isolated sub-network of the pathway as opposed to computing pathway's perturbation status using enrichment statistics. The name DPA (Deep Pathway Analyzer) originates from the novelty of our method that can deeply recognize perturbed portions of the pathways. Our method of programmatically identifying "localized" regulating portion of the pathways could pave a new way to carry out future pathway analysis.

\section{Abbreviations}

AUC: Area Under Curve; DE genes: Differentially Expressed genes; DPA: Deep Pathway Analyzer; KEGG: Kyoto Encyclopedia of Genes and Genomes; ROC curve: Receiver operating characteristic curve

\section{Acknowledgements}

The authors are grateful to Charles Giardina for his help in interpreting the outcomes from their automated pathway analysis method. They also thank NVIDIA who donated the Titan Xp which has been extensively used in this research. 


\section{About this supplement}

This article has been published as part of BMC Genomics, Volume 21 Supplement 1, 2020: Selected articles from the 14th International Symposium on Bioinformatics Research and Applications (ISBRA-18): genomics. The full contents of the supplement are available at https://bmcgenomics. biomedcentral.com/articles/supplements/volume-21-supplement-1.

\section{Authors' contributions}

YZ designed algorithms, performed theoretical proof, implemented the whole framework, carried out experiments, performed analysis and wrote the manuscript. SP helped with result interpretation and TH did experiment analysis. DGS helped design the algorithms, refined the manuscript and supervised the project. All authors have read and approved the final manuscript.

\section{Funding}

This work was supported in part by National Institutes of Health, Grant No. HD098636 to Dong-Guk Shin. Yue Zhao's work was supported in part by the pre-doctoral fellowship by the Department of Computer Science and Engineering, University of Connecticut. National Institute of Child Health and Human Development had no role in designing the study, collecting and analyzing data, or preparing the manuscript.

\section{Availability of data and materials}

All data analyzed during this study are included in this article.

\section{Ethics approval and consent to participate}

Not applicable.

\section{Consent of publication}

Not applicable.

\section{Competing interests}

The authors declare that they have no competing interests.

\section{Author details}

${ }^{1}$ Computer Science and Engineering Department, University of Connecticut, 371 Fairfield Way, Unit 4155, 06269 Storrs, USA. ${ }^{2}$ Department of Pharmaceutical Sciences, University of Connecticut, 69 North Eagleville Road, Unit 3092, Storrs, USA.

\section{Received: 11 September 2019 Accepted: 30 September 2019} Published: 5 March 2020

\section{References}

1. Ogata H, Goto S, Sato K, Fujibuchi W, Bono H, Kanehisa M. Kegg: Kyoto encyclopedia of genes and genomes. Nucleic Acids Res. 1999;27(1):29-34

2. Nishimura D. Biotech Software \& Internet Report: The Computer Software Journal for Scient. 2001;2(3):117-20.

3. Croft D, Mundo AF, Haw R, Milacic M, Weiser J, Wu G, Caudy M, Garapati P, Gillespie M, Kamdar MR, et al. The reactome pathway knowledgebase. Nucleic Acids Res. 2013;42(D1):472-7.

4. Kutmon M, Riutta A, Nunes N, Hanspers K, Willighagen EL, Bohler A, Mélius J, Waagmeester A, Sinha SR, Miller R, et al. Wikipathways: capturing the full diversity of pathway knowledge. Nucleic Acids Res. 2015;44(D1):488-94.

5. Ritchie ME, Phipson B, Wu D, Hu Y, Law CW, Shi W, Smyth GK. limma powers differential expression analyses for rna-sequencing and microarray studies. Nucleic Acids Res. 2015;43(7):47.

6. Li J, Tibshirani R. Finding consistent patterns: a nonparametric approach for identifying differential expression in rna-seq data. Stat Methods Med Res. 2013;22(5):519-36.

7. Ghosh D, Chinnaiyan AM. Mixture modelling of gene expression data from microarray experiments. Bioinformatics. 2002;18(2):275-86.

8. Babicki S, Arndt D, Marcu A, Liang Y, Grant JR, Maciejewski A, Wishart DS. Heatmapper: web-enabled heat mapping for all. Nucleic Acids Res. 2016;44(W1):147-53.

9. Joshi P, Pei B, Hong S-H, Kalajzic I, Shin D-J, Rowe D, Shin D-G. A software framework integrating gene expression patterns, binding site analysis and gene ontology to hypothesize gene regulation relationships. In: Bioinformatics and Biomedicine (BIBM), 2013 IEEE International
Conference On. IEEE; 2013. p. 210-213. https://doi.org/10.1109/bibm. 2013.6732491.

10. Shin D-G, Hong S-H, Joshi P, Nori R, Pei B, Wang H-W, Harrington P, Kuo L, Kalajzic I, Rowe D. Pbc: A software framework facilitating pattern-based clustering for microarray data analysis. In: Bioinformatics, Systems Biology and Intelligent Computing, 2009. IJCBS'09. International Joint Conference On. IEEE; 2009. p. 30-6. https://doi.org/10.1109/ijcbs.2009.113.

11. Falcon $S$, Gentleman R. Using gostats to test gene lists for go term association. Bioinformatics. 2006;23(2):257-8.

12. Huang DW, Sherman BT, Tan Q, Collins JR, Alvord WG, Roayaei J, Stephens R, Baseler MW, Lane HC, Lempicki RA. The david gene functional classification tool: a novel biological module-centric algorithm to functionally analyze large gene lists. Genome Biol. 2007;8(9):183.

13. Subramanian A, Tamayo P, Mootha VK, Mukherjee S, Ebert BL, Gillette MA, Paulovich A, Pomeroy SL, Golub TR, Lander ES, et al. Gene set enrichment analysis: a knowledge-based approach for interpreting genome-wide expression profiles. Proc Natl Acad Sci. 2005;102(43):15545-50.

14. Vaske CJ, Benz SC, Sanborn JZ, Earl D, Szeto C, Zhu J, Haussler D, Stuart JM. Inference of patient-specific pathway activities from multi-dimensional cancer genomics data using paradigm. Bioinformatics. 2010;26(12): 237-45.

15. Tarca AL, Draghici S, Khatri P, Hassan SS, Mittal P, Kim J-S, Kim CJ, Kusanovic JP, Romero R. A novel signaling pathway impact analysis. Bioinformatics. 2009;25(1):75-82.

16. Shin D-G, Kazmi SA, Pei B, Kim Y-A, Maddox J, Nori R, Wong A, Krueger W, Rowe D. Computing consistency between microarray data and known gene regulation relationships. IEEE Trans Inf Technol Biomed. 2009;13(6): 1075-82.

17. Zhao Y, Chen M-H, Pei B, Rowe D, Shin D-G, Xie W, Yu F, Kuo L. A bayesian approach to pathway analysis by integrating gene-gene functional directions and microarray data. Stat Biosci. 2012;4(1):105-31.

18. Zhao $Y$, Hoang $T H$, Joshi $P$, Hong $S-H$, Shin D-G. Deep pathway analysis incorporating mutation information and gene expression data. In: Bioinformatics and Biomedicine (BIBM), 2016 IEEE International Conference On. IEEE; 2016. p. 260-265. https://doi.org/10.1109/bibm. 2016.7822528.

19. Zhao Y, Hoang TH, Joshi P, Hong S-H, Giardina C, Shin D-G. A route-based pathway analysis framework integrating mutation information and gene expression data. Methods. 2017;124:3-12. https:// doi.org/10.1016/j.ymeth.2017.06.016.

20. Korucuoglu M, Isci S, Ozgur A, Otu HH. Bayesian pathway analysis of cancer microarray data. PloS ONE. 2014;9(7):102803.

21. Isci S, Ozturk C, Jones J, Otu HH. Pathway analysis of high-throughput biological data within a bayesian network framework. Bioinformatics. 2011;27(12):1667-74.

22. Ma S, Jiang T, Jiang R. Differential regulation enrichment analysis via the integration of transcriptional regulatory network and gene expression data. Bioinformatics. 2014;31(4):563-71.

23. Kaushik A, Ali S, Gupta D. Altered pathway analyzer: A gene expression dataset analysis tool for identification and prioritization of differentially regulated and network rewired pathways. Sci Rep. 2017;7:40450.

24. Whitfield ML, Sherlock G, Saldanha AJ, Murray JI, Ball CA, Alexander KE, Matese JC, Perou CM, Hurt MM, Brown PO, et al. Identification of genes periodically expressed in the human cell cycle and their expression in tumors. Mol Biol Cell. 2002;13(6):1977-2000.

25. Wang Y. H. On the number of successes in independent trials. Stat Sin. 1993;3:295-312.

26. Choi K, Xia A. Approximating the number of successes in independent trials: Binomial versus poisson. Ann Appl Probab. 2002;12:1139-1148. https://doi.org/10.1214/aoap/1037125856

27. Feller W. On the normal approximation to the binomial distribution. In: Selected Papers I. Springer; 2015. p. 655-65. https://doi.org/10.1007/9783-319-16859-3_32.

28. Cormen T, Leiserson C, Rivest R, Stein C. Introduction to Algorithms, 2nd. Cambridge, MA: The MIT Press and McGraw-Hill Book Company; 2001, pp. 540-9.

29. Sherlock G, Hernandez-Boussard T, Kasarskis A, Binkley G, Matese JC, Dwight SS, Kaloper M, Weng S, Jin H, Ball CA, et al. The stanford microarray database. Nucleic Acids Res. 2001;29(1):152-5.

30. Gentry J, Long L, Gentleman R, Falcon S, Hahne F, Sarkar D, Rgraphviz KH. Provides plotting capabilities for $r$ graph objects. R Packag version. 2009;2(0):. 
31. Cho RJ, Huang M, Campbell MJ, Dong H., Steinmetz L, Sapinoso L, Hampton G, Elledge SJ, Davis RW, Lockhart DJ. Transcriptional regulation and function during the human cell cycle. Nat Genet. 2001;27(1):48.

32. Zambon AC, Zhang L, Minovitsky S, Kanter JR, Prabhakar S, Salomonis N, Vranizan K, Dubchak I, Conklin BR, Insel PA. Proc Natl Acad Sci U S A. 2005;102(24):8561-66.

33. Friedman J, Hastie T, Tibshirani R. Regularization paths for generalized linear models via coordinate descent. J Stat Softw. 2010;33(1):1-22.

34. Olivier M, Eeles R, Hollstein M, Khan MA, Harris CC, Hainaut P. The iarc tp53 database: new online mutation analysis and recommendations to users. Human mutation. 2002;19(6):607-14.

35. Huang DW, Sherman BT, Lempicki RA. Bioinformatics enrichment tools: paths toward the comprehensive functional analysis of large gene lists. Nucleic Acids Res. 2008;37(1):1-13.

36. Cho SB, Kim J, Kim JH. Identifying set-wise differential co-expression in gene expression microarray data. BMC bioinformatics. 2009;10(1):109.

37. Rahmatallah Y, Emmert-Streib F, Glazko G. Gene sets net correlations analysis (gsnca): a multivariate differential coexpression test for gene sets. Bioinformatics. 2013;30(3):360-8.

38. Han J, Shi X, Zhang Y, Xu Y, Jiang Y, Zhang C, Feng L, Yang H, Shang D, Sun Z, et al. Esea: discovering the dysregulated pathways based on edge set enrichment analysis. Sci Rep. 2015;5:13044.

39. Hung J-H, Whitfield TW, Yang T-H, Hu Z, Weng Z, DeLisi C. Identification of functional modules that correlate with phenotypic difference: the influence of network topology. Genome Biol. 2010;11(2):23.

40. Fischer M. Census and evaluation of p53 target genes. Oncogene. 2017;36(28):3943.

41. Ruxton GD. The unequal variance t-test is an underused alternative to student's t-test and the mann-whitney u test. Behav Ecol. 2006;17(4): 688-90.

\section{Publisher's Note}

Springer Nature remains neutral with regard to jurisdictional claims in published maps and institutional affiliations.

Ready to submit your research? Choose BMC and benefit from:

- fast, convenient online submission

- thorough peer review by experienced researchers in your field

- rapid publication on acceptance

- support for research data, including large and complex data types

- gold Open Access which fosters wider collaboration and increased citations

- maximum visibility for your research: over $100 \mathrm{M}$ website views per year

At BMC, research is always in progress.

Learn more biomedcentral.com/submissions 